\title{
Parallel Alignment of a Large Number of Range Images
}

\author{
Takeshi Oishi \\ Institute of Industrial Science, \\ The University of Tokyo \\ oishi@cvl.iis.u-tokyo.ac.jp \\ Atsushi Nakazawa \\ Osaka University \\ nakazawa@ime.cmc.osaka-u.ac.jp \\ Ryusuke Sagawa \\ Osaka University \\ sagawa@am.sanken.osaka-u.ac.jp \\ Katsushi Ikeuchi \\ Institute of Industrial Science, The University of Tokyo \\ ki@cvl.iis.u-tokyo.ac.jp
}

\begin{abstract}
This paper describes a method for parallel alignment of multiple range images. It is difficult to align a large number of range images simultaneously. Therefore, we developed the parallel method to improve the time and memory performances of the alignment process. Although a general simultaneous alignment algorithm searches correspondences for all pairs of all range images by rejecting redundant dependencies, our method makes it possible to accelerate computation time and reduce the amount of memory used. Since the computation between two range images can be preformed independently, each correspondence pair of range images is assigned to each node. Because the computation time is proportional to the number of vertices assigned to each node, by assigning the pairs so that the number of vertices computed is equal on each node, the load on each node is effectively distributed. The heuristic algorithms for graph partitioning are applied to this problem in order to reduce the amount of memory used on each node. The method was tested on a 16 processor PC cluster, where it demonstrated the high extendibility and the performance improvement in time and memory.
\end{abstract}

\section{Introduction}

In recent years, the technology of modeling from reality has been highly developed [1]. Using this technology has enabled the digital preservation of many precious cultural heritage objects around the world [2,3]. The laser range finders used for digital preservation can measure a $3 \mathrm{D}$ surface geometry with accuracy within a millimeter for a short distance. For a long distance (50m to $100 \mathrm{~m})$, a laser range finder that applies a time of flight can obtain surface geometry with 1 centimeter degree of accuracy.
While the performance of laser range finders has been greatly improved, there are still some problems in creating a 3D model from a large number of range images. Because a laser range finder can measure only the visible surface, it is necessary to take range images from many different directions, and some post-processes are required in order to obtain a complete 3D model. Once the scanning has been completed, all range images are aligned into a common coordinate system. If an object is small enough to be put on a turntable, it is easy to obtain the relative positions of the range images. But in cases where an object is a large statue, for example, it is difficult to record the accurate position and direction of the laser range finder. Therefore, a computation to obtain the relative positions of the range images is required. (If a large number of range images are aligned, computation time and the amount of memory used become very large. That is one of the problems encountered in creating 3D models from a large number of range images.) After the range image positions are acquired, they are merged into a unified mesh model. Turk et al. proposed a method which zippers range images together [4]. In $[5,6]$, methods that use volumetric representation in order to achieve data merging are proposed. To handle a large number of range images, as one of the solutions, a parallel merging algorithm [7] is developed by Sagawa et al. Then we describe how we develop a method to align a large number of range images.

Many methods of aligning range images are based on the iterative closest point (ICP) proposed by Besl [8] and are adapted from the method proposed by Chen [9]. With ICP, correspondences are searched for as the closest points between two range images, and a transformation matrix is computed so that the mean square error of the correspondences is minimized. The computation is 
iterated until the mean square error falls below the threshold value. On the other hand, in Chen's method, the relative positions of range images are calculated so that the distance between vertices and the corresponding meshes is minimized. In addition, there is a method to search for correspondences by projecting the points along with the ray direction [10]. Moreover, since the ICP algorithm tends to be affected by false matching and noise, Masuda et al. proposed a more robust method that uses random sampling and Least Median Squares Estimation (LMedS) [11].

The algorithms described above align two range images; however, when using these algorithms, error accumulation increases as the number of range images increases. In such cases, a method that simultaneously aligns a large number of range images is required. Neugebauer et al. proposed a simultaneous registration method that adopted projection search of correspondences and point-plane error evaluation [12]. This is the fundamental algorithm used in this study. Benjamaa et al. extended the method proposed by Bergevin et al. [13] and implemented a simultaneous alignment method while they accelerated the pair-wise alignment algorithm by using multi z-buffers [14].

Thus, although various methods have been proposed, the problem for every method is the computation cost of correspondence search. If the number of vertices of two range images is equally assumed to be $n$ by the original ICP, their complexity is $O\left(n^{2}\right)$ since correspondences are searched for in all vertices. In addition, there are techniques [15] that use kd-trees and that narrow the search range by using the data cache [16] in order to accelerate ICP, as well as the parallel ICP algorithm proposed by Langis et al., which is implemented on a PC cluster [17].

Despite the many alignment algorithms, it is difficult to simultaneously align the large number of range images that we treat. Because the calculation time for the pairs of range images increases, and it is necessary to read all range images into memory when such algorithms are used (Parallel ICP [17] does not consider the amount of memory used). It is thought, moreover, that the amount of data will increase along with the development of measurement technology. Therefore, we need a method in which the calculation time is short, the amount of memory used is small, and the extendibility is high. Thus, we propose a parallel simultaneous alignment method that is implemented on a PC cluster; this method is cheap and has high extendibility.

In Section 2, the fundamental alignment algorithm is described. In Section 3, we present the algorithm of parallel computation. Sections 4 and 5 contain the evaluations of this algorithm and the alignment results of a large number of range images, respectively. Our conclusions are presented in Section 6.

\section{Alignment Algorithm}

In this section, the outline of the fundamental alignment algorithm is explained. The procedures of the algorithm are as follows.

1. Search correspondences for all the vertices of the combination of all range images.

2. Calculate the errors between corresponding points.

3. Calculate transformation matrices that minimize the errors of the correspondences.

4. Reiterate Steps1-3 until end conditions are fulfilled. Details of search corresponding points, error evaluation, and calculation of transformation matrices are explained below.

\subsection{Search corresponding points}

While ICP searches the closest points as corresponding points, this method searches for corresponding points along the line of sight. Assume that a base range image is the model image and that a corresponding range image is the scene image. In Figure 1, a model image is depicted as points and a scene image is shown as meshes. When a line extended from a vertex of model image along the line of sight crosses a mesh of a scene image, the intersecting point is the corresponding point. In order to eliminate wrong correspondences, if the distance between corresponding points is larger than a threshold distance and if the angle of lines of sight is beyond a threshold angle, the correspondence is rejected. This correspondence search is computed for every pair of range images.

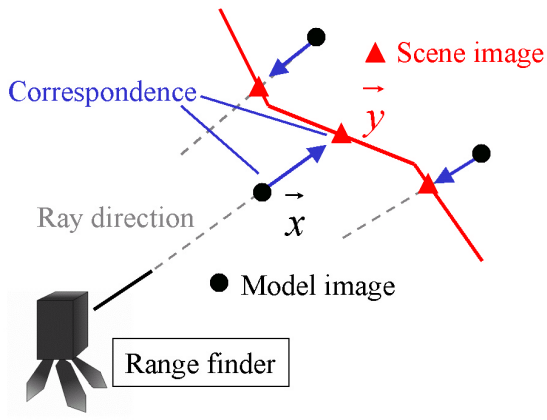

Figure 1. Search corresponding points

\subsection{Error evaluation}

The error between corresponding points is the length of the point and the plane. Let the vertex of model image be $\boldsymbol{x}$ and let the point corresponding to the vertex be $\boldsymbol{y}$; the error between the corresponding points is written as

$$
\boldsymbol{n} \cdot(\boldsymbol{y}-\boldsymbol{x})
$$

where $\boldsymbol{n}$ is the vertex normal of $\boldsymbol{x}$, and this vector is computed previously. The transformation matrices of the model and scene images are computed so that the error is minimized. The error evaluation function is rewritten as

$$
\boldsymbol{R}_{M} \boldsymbol{n} \cdot\left\{\left(\boldsymbol{R}_{S} \boldsymbol{y}+\boldsymbol{t}_{S}\right)-\left(\boldsymbol{R}_{M} \boldsymbol{x}+\boldsymbol{t}_{M}\right)\right\}
$$

The rotation matrix and the translation vector of the model 
image are $\boldsymbol{R}_{M}$ and $\boldsymbol{t}_{M}$, respectively. The rotation matrix and the translation vector of the scene image are $\boldsymbol{R}_{S}$ and $\boldsymbol{t}_{S}$, respectively. Then, the distance between the $i$ th range image and the $j$ th image is expressed for every vertex $k$ as follows.

$$
\varepsilon^{2}=\min _{\boldsymbol{R}, t} \sum_{i \neq j, k}\left(\boldsymbol{R}_{M} \boldsymbol{n} \cdot\left\{\left(\boldsymbol{R}_{S} \boldsymbol{y}+\boldsymbol{t}_{S}\right)-\left(\boldsymbol{R}_{M} \boldsymbol{x}+\boldsymbol{t}_{M}\right)\right\}\right)^{2}
$$

\subsection{Compute transformation}

If it is assumed that the angles of rotation are minute, the rotation matrix $\boldsymbol{R}$ is written as

$$
\boldsymbol{R}=\left(\begin{array}{ccc}
1 & -c_{3} & c_{2} \\
c_{3} & 1 & -c_{1} \\
-c_{2} & c_{1} & 1
\end{array}\right)
$$

The translation vector is expressed as

$$
\boldsymbol{t}=\left(\begin{array}{lll}
t_{x} & t_{y} & t_{z}
\end{array}\right)
$$

After some algebraic manipulations [14], (3) is rewritten as

$$
\begin{gathered}
\varepsilon^{2}=\min _{\delta} \sum_{i \neq j, k}\left\|\boldsymbol{A}_{i j k} \boldsymbol{\delta}-\boldsymbol{s}_{i j k}\right\|^{2} \\
\boldsymbol{s}_{i j k}=\boldsymbol{n}_{i k} \cdot\left(\boldsymbol{x}_{i k}-\boldsymbol{y}_{i j k}\right) \\
\boldsymbol{A}_{i j k}=(\underbrace{0 \ldots 0}_{6 i \times 1} \underbrace{\boldsymbol{C}_{i j k}}_{6 \times 1} \underbrace{0 \ldots 0}_{6(l-i-1) \times 1})+(\underbrace{0 \ldots 0}_{6 j \times 1} \underbrace{0-\boldsymbol{C}_{i j k}}_{6 \times 1} \underbrace{0 \ldots 0}_{6(l-j-1) \times 1}) \\
\boldsymbol{C}_{i j k}=\left(\begin{array}{c}
\boldsymbol{n}_{i k} \times \boldsymbol{y}_{i j k} \\
-\boldsymbol{n}_{i k}
\end{array}\right) \\
\boldsymbol{\delta}=\left(\boldsymbol{m}_{0} \cdots \boldsymbol{m}_{n-1}\right) \\
\boldsymbol{m}_{i}=\left(\begin{array}{lll}
c_{1 i} & c_{2 i} & c_{3 i} \\
t_{x i} & t_{y i} \quad t_{z i}
\end{array}\right)
\end{gathered}
$$

where the number of range images is $n$. By (6) $\delta$ is written as

$$
\boldsymbol{\delta}=\left(\sum_{i \neq j, k} \boldsymbol{A}_{i j k}^{T} \boldsymbol{A}_{i j k}\right)^{-1} \sum_{i \neq j, k} \boldsymbol{A}_{i j k}^{T} \boldsymbol{S}_{i j k}
$$

\section{Parallelization of simultaneous alignment}

Since the computation of error matrices $\boldsymbol{A}^{T}{ }_{i j k} \boldsymbol{A}_{i j k}$ and $\boldsymbol{A}^{T}{ }_{i j k} \boldsymbol{S}_{i j k}$ between two range images can be performed independently, correspondence pairs of range images are assigned to each node. We implemented our method as a server/client system. The server program holds transformation matrices from initial position to current position of all range images. First, server program checks all pairs, and creates the list of computations for each node. The client program receives the list of pairs and reads the required range images into memory. Then, each client computes the error matrices independently, and sends the matrices to the server program. The server program computes the transformation matrices of all range images from the error matrices received from the client program. The results are applied to all server/client data. Then, each iteration process is continued until the error falls below threshold value. The pseudo code of the procedures is as follows:

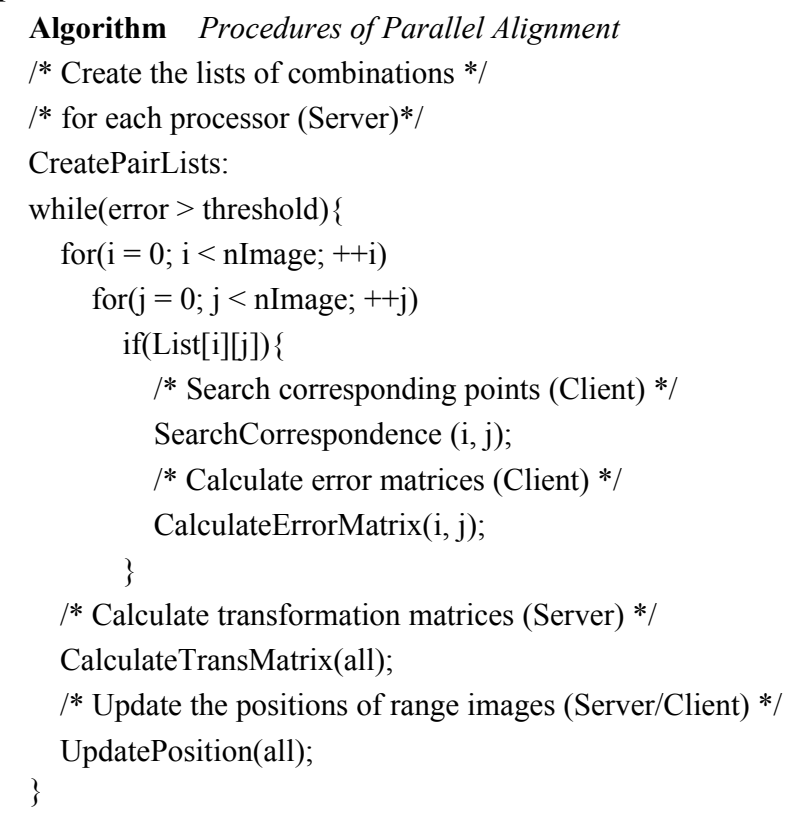

The list of pairs computed by each client is created once at the beginning of iterations, so the computation time and the amount of memory used by each client do not change at each iteration.

\subsection{Removing redundant dependencies}

When the alignment algorithm is parallelized, both time performance and memory performance have to be considered. The simultaneous alignment algorithm, originally designed, requires all range images to be read into memory; even when the computation is distributed over nodes, the amount of memory used on each node is not reduced.

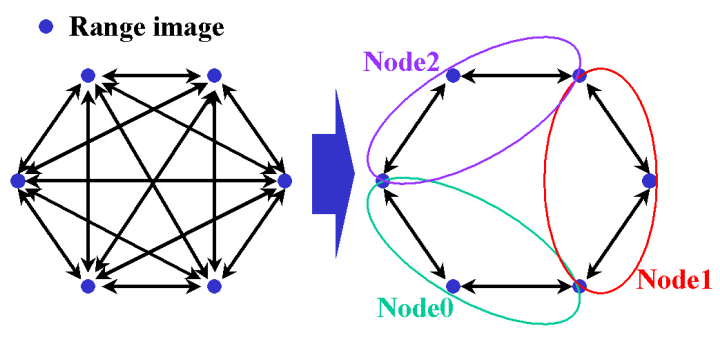

Figure 2. Remove redundant dependencies

However, if the number of pairs that have to be computed can be decreased in advance, it is possible to reduce data size read into each node. There are many redundant pairs in the relationship of all range images. If there is no overlapping region between two range images or if the overlapped region is small, the pair of these range images does not affect the estimation of the positions. 
Then, by removing such pairs, time performance is accelerated and the amount of memory used in each node can be reduced as shown in Figure 2.

\subsubsection{Rejection by global information}

We can define and remove redundant dependencies in the range images. A pair of range images that does not satisfy the following requirements will be removed as redundant relations.

1. The bounding-boxes of two range images overlap each other.

2. The angle $\theta$ between rays of two range images is less than a threshold value.

Under the assumption that initial positions of two range images are accurately estimated, Requirement 1 is satisfied when a sufficient overlapped region exists between two range images. Requirement 2 is satisfied when two views are relatively near. As a side effect, this requirement also reduces the possibility of false correspondences. Since our method utilizes the ray direction to establish correspondences, by setting the threshold, as $\theta=90^{\circ}$, we can avoid correspondence pairs between one and the other side.

\subsubsection{Local overlapping area}

Next, the overlapping area between two range images is calculated for all remained pairs. The overlapping area is expressed as the ratio of the number of vertices included in one range image and the number of corresponding points between that image and another image. However, as mentioned above, since searching for corresponding points is time consuming, corresponding points are searched for a few vertices selected randomly. We used $10 \%$ of the vertices for this search. Let the number of vertices selected randomly in range image $i$ be $v_{i}^{\prime}$, and let the number of corresponding points between range images $i$ and $j$ be $v_{c i, j}^{\prime}$, the overlapping area $S_{i, j}$ between the range images is written as follows.

$$
S_{i, j}=v_{c i, j}^{\prime} / v_{i}^{\prime}
$$

A pair whose overlapping area $S_{i, j}$ is less than threshold value will be removed as weak data dependency. We set the threshold value as 0.03 to 0.05 . Of course, since the computation of overlapping areas can be performed independently and sequentially, the computations are performed easily in parallel without the problem of memory space.

\subsubsection{Adjacent relation}

The last requirement is set up for removing non-adjacent relations sequentially. For example, as shown in Figure 3, if the length from $I_{0}$ to $I_{n}$ is larger than the length from $I_{m}$ to $I_{n} \quad\left(l_{0 m}<l_{0 n}\right)$, the arc between $I_{0}$ and $I_{n}$ is removed. Here, the distance is evaluated from the center of a range image's surface. After the removing process by this requirement, since the number of pairs for a range image becomes 12 or less, with the number of range images $m$, the calculation complexity is reduced to $O(12 \mathrm{~m})$ from $O\left(\mathrm{~m}^{2}\right)$. And error accumulation is distributed to different directions.

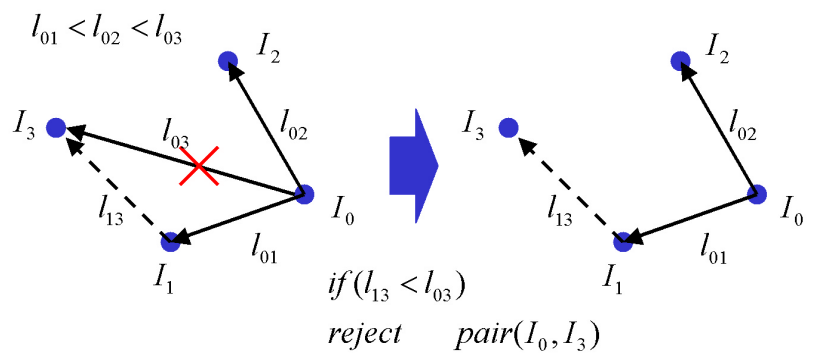

Figure 3. Rejecting pairs by relative distances

\subsection{Parallelization by graph partitioning}

The problem of load balancing with minimum amount of memory used is an NP-hard problem [18]. Therefore, it is difficult to obtain the optimal solution in a reasonable amount of time. In that case, we propose a method to solve this problem by applying the heuristic algorithms for graph partitioning.

\subsubsection{Pair-node hypergraph}

First, we define the pair-node hypergraph. The left image of Figure 4 shows the graph expressed the relations of the range images. A new graph in which each node expresses pairs $P_{i, j}$ between two range images $i$ and $j$ is created from the original graph as shown in Figure 4. The created graph is the hypergraph in which all nodes are connected to two nets. We call it a pair-node hypergraph. The node weight $W_{i, j}^{\text {node }}$ is equal to the sum of the number of vertices $v_{i}$ and $v_{j}$, and the net weight $W^{n e t}{ }_{i}$ is equal to the number of vertices $v_{i}$.

$$
\begin{aligned}
& W_{i, j}^{\text {node }}=v_{i}+v_{j} \\
& W_{i}^{\text {net }}=v_{i}
\end{aligned}
$$

The pair-node hypergraph is partitioned such that the sum of the node-weight in each subset is the same and the sum of the net-weight included in each subset is minimized.

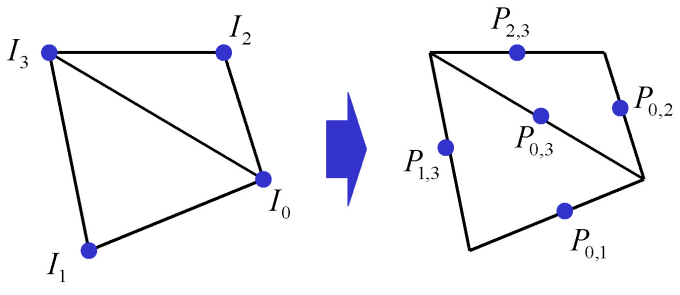

Figure 4. Correspondence pair node hyper-graph

\subsubsection{Initial partitioning}

The pair-node hypergraph is initially partitioned so that the sum of the node-weight in each subset is the same. Spectral bisection methods $[19,20]$ that minimize the edge-cut by using second eigenvector is widely available, but it is difficult to apply the method to our problem. Intelligent graph growth algorithm [21] can obtain fairly optimal solution in small computation time. However, this 
method tends to be trapped in a poor partitioning [22]. Then, we used the random seeded breadth first search method for initial partitioning [22, 23]. This method is simple but can generate better results. The graph growth bisection method may create an isolated partition as shown in Figure 5 (a). In that case, partitions are grown again by reverse propagation as shown in Figure 5 (b) and (c). Since the sum of net-weight included in subsets is greatly influenced by the selection of the seed, we tried to create initial partitions for several seeds and adopted the partition in which the sum of net-weight included is minimum. In order to obtain k-way partitions, the recursive bisection method is used. After $\log k$ phases, the graph is partitioned into $k$ parts [23].

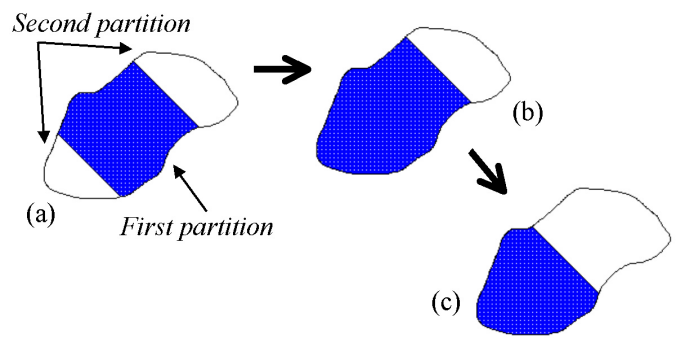

Figure 5. Reverse propagation

\subsubsection{Refine partitions}

The partitioned graph is refined so that the sum of net-weight included in subsets is minimized. The method to refine bisected partition is proposed by Kernighan and Lin [24]. The FM (Fiduccia and Mattheyses) algorithm [25] develops several improvements to the original KL algorithm and reduces complexity. These methods are an iterative improvement algorithm by nature. The algorithm moves a node from a partition to another so that the operation to move the node causes the greatest improvement in the cutsize.

We improved the KLFM algorithm so that the refinement process minimizes the sum of net-weight included in subsets of the graph. Original KLFM algorithm moves a node at a time, but our method moves a net at a time. That is, all nodes connected to the net are moved at a time. This method is similar to the algorithm that has lookahead ability [26]. Like the clustering method and the multi-level method, this method climbs out of local minima. For k-way refinement, the partition of which the sum of net-weight is maximum weight is calculated with all remaining partitions. The refinement process is iterated until there is no more improvement.

The net gain is calculated for all nets that are in the frontier of subset graphs. In the case that a net $N_{(i, j), k}$ connected to subset graphs $G_{i}$ and $G_{j}$ is moved to $G_{i}$, the gain $g_{i j, k}$ is expressed by $D^{\text {int }}{ }_{i, j, k}$; that means the variation of the sum of net weight of $G_{i}$ and $D^{\text {ext }}{ }_{i, j, k}$ that means the variation of the sum of net-weight of $G_{i}$.

$$
g_{i, j, k}=D^{\mathrm{int}}{ }_{i, j, k}-D^{e x t}{ }_{i, j, k}
$$

On the other hand, in the case where $N_{(i, j), k}$ is moved to

$G_{j}$, the gain $g_{j, i, k}$ is expressed by internal degree $D^{l_{\text {int }}{ }_{j, i, k}}$ and external degree $D^{\text {ext }}{ }_{j, i, k}$.

$$
g_{j, i, k}=D_{j, i, k}^{\mathrm{int}}-D_{j, i, k}^{e x t}
$$

Two lists are created for two gains. A net that has the smallest gain is selected from one of the lists and moved. The list that the net has selected has a greater size of the sum of node-weight than another list. When a net is moved, all nets and nodes concerned with the net are updated, and the moved net is locked in order to avoid thrashing. The sum of net-weight and moved net's id are recorded at each movement. After one path has been performed, the minimum value recorded in that path is compared with the value of current sum of net-weight. If the minimum value is smaller than that of current state, refinement is achieved, and the refinement process is iterated. If not, refinement process is terminated.

\section{Performance evaluation}

This method was implemented on a PC cluster that consisted of 8 PCs. Each PC had dual ArhlonMP2400+ processors and 4Gbytes of memory, and was connected by 100Base-TX ether net. The range images used for evaluation were 50 images created artificially from the complete 3D model of the Great Buddha of Kamakura. Figure 6 shows the original 3D model of the statue and the range images created artificially. These range images contain an average of 83,288 vertices and 158,376 meshes. In this section, our method is evaluated from the point of convergence and accuracy, computation time, and memory performance.

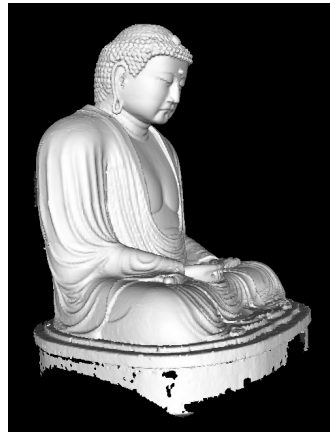

(a) Original model Figure 6. Range images for evaluations

\subsection{Convergence and accuracy}

Because our method rejects the redundant dependencies, the influence of the rejection to convergence and accuracy has to be evaluated. In the case of these range images, the number of all pairs is 2,450 , but it is reduced to 160 by the rejection process. It is verified whether accurate convergence is performed even when the number of pairs becomes small. Virtually created range images have accurate positions of measured points, so convergence and accuracy of alignment can be evaluated by the distance 
between accurately aligned range image and the target range image. The distance between two range images is defined as an average of the euclidean length of all vertices. Each range image added Gaussian noise along the line of sight at maximum length $10 \mathrm{~mm}$. All range images were moved at random in the maximum length of $100 \mathrm{~mm}$ in the direction of $\mathrm{x}, \mathrm{y}, \mathrm{z}$, respectively, and rotated at random in the maximum angle of 0.05 radian to the $\mathrm{x}$-axis, $\mathrm{y}$-axis, and $\mathrm{z}$-axis, respectively.

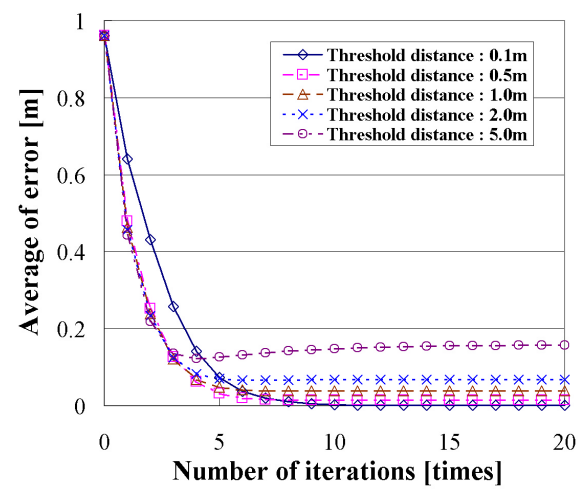

Figure 7. Convergence with original method

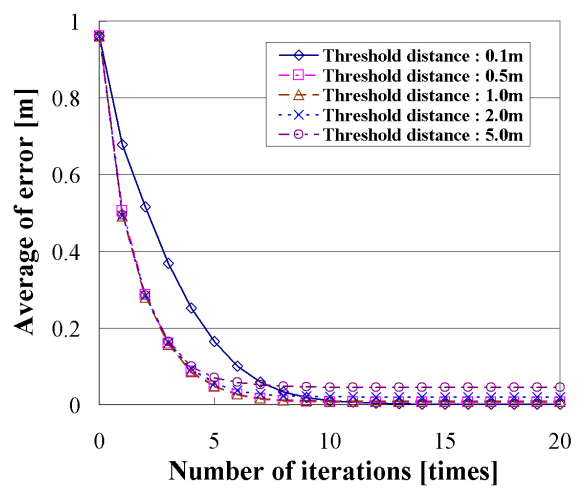

Figure 8. Convergence with our method

The results of the original method and our method are shown in Figures 7 and 8, respectively. The threshold distance for rejecting outliers while searching for correspondences is changed gradually. Although both the original method and our method do not converge at the correct positions when the threshold distance is $5 \mathrm{~m}$, our method converges at a better position than the position of the original method. Although the convergence speed of our method is slower than that of the original method, our method tends to converge at the better position than the position of the original method. It is thought that this is because the rejection of redundant pairs reduces false correspondence of range images. The alignment algorithm that we used has the feature that it tends to be influenced by false correspondence and noise. Therefore, by rejecting redundant range images, transformations are accurately estimated. When the threshold values are $0.1 \mathrm{~m}$ and $0.5 \mathrm{~m}$, the error converges at approximately 0 . It turns out that the accurate estimation is acquired by our method.

\subsection{Time performance}

Here, the time performance is evaluated. Computation time is defined as the time taken for one iteration, and an average of time of all iterations is used for the evaluation. Figure 9 shows the time ratio with the number of processors. Computation time $T_{n}$ is expressed as the ratio to the computation time with one processor $T_{0}$. This figure shows that the computation time is linearly improved as the number of processors increases. Moreover, our method improves computation time unlike the sequential method in which the range images are assigned in arbitrary selected order. An actual computation time with one processor is an average of $20560 \mathrm{~ms}$, and the computation time with 16 processors is an average of $1784 \mathrm{~ms}$. The computation time with 16 processors is approximately 11.5 times faster than that with 1 processor.

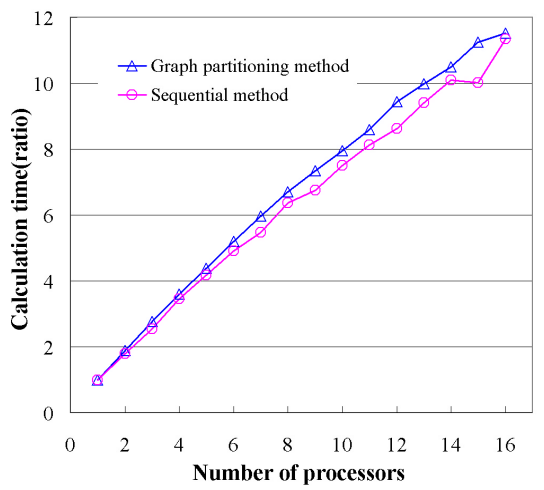

Figure 9. Computational Efficiency

\subsection{Amount of memory used}

Next, the evaluation of memory performance is shown. The amount of memory used is shown in Figure 10 with the number of processors. Each value shows the ratio with the amount of memory used with single processor. It appears that the amount of required memory decreases as the number of processors increases. Compared with the sequential method, the performance is highly improved by our method. An actual maximum size of required memory with one processor is 269Mbytes and that with 16 processors is $48 \mathrm{Mbytes}$. It turns out that our method could reduce the amount of memory used by approximately $17 \%$ for these range images.

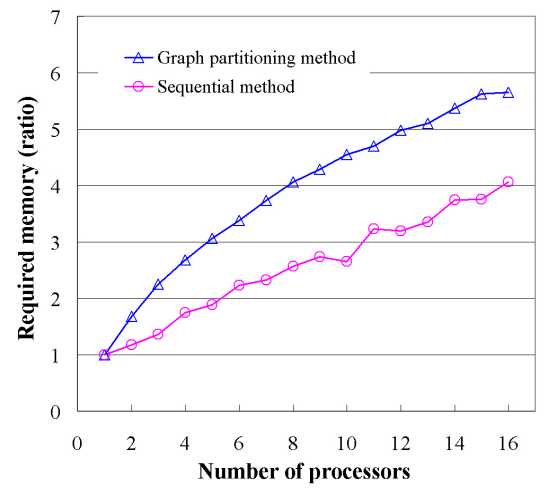

Figure 10. Required memory 


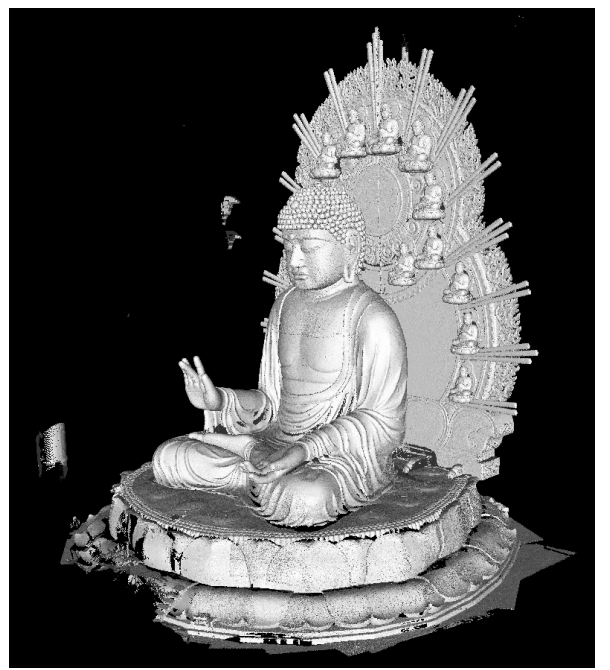

Figure 11. Alignment result (Nara Buddha)

\section{Experimental results}

In this section, we will show the result of parallel alignment of a large number of range images that could not be aligned because of limitation of memory space. We used the following two sets of range images.

Model-1. 114 range images that measured the Great Buddha of Nara by Cyrax2400 [27] (contain an average of 327,470 vertices and 606,072 meshes)

Model-2. 210 range images that measured the Bayon in Cambodia by Cyrax2500 (contain an average of 433,785 vertices and 798,890 meshes)

The minimum parallelization numbers that the maximum amount of required memory is under the limitation are 2 and 4 for Model-1 and Model-2, respectively. The alignment results computed by the minimum number of processors and the maximum number of processors (16) are shown in Table 1 and Table 2. These tables show the computation time and maximum amount of memory used and minimum amount of memory used.

Table 1. Total performance (Nara)

\begin{tabular}{|c|c|c|c|}
\hline Processor & Time(s) & Max Mem(MB) & Min Mem(MB) \\
\hline 2 & 76.0 & 1287 & 1275 \\
\hline 16 & 13.2 & 292 & 254 \\
\hline
\end{tabular}

Table 2. Total performance (Bayon)

\begin{tabular}{|c|c|c|c|}
\hline Processor & Time(s) & Max Mem(MB) & Min Mem(MB) \\
\hline 4 & 103.9 & 1608 & 1456 \\
\hline 16 & 40.2 & 559 & 472 \\
\hline
\end{tabular}

In the case of Model-1, the computation time is improved 5.75 times faster than that with 2 processors, and amount of memory used is reduced in $22.6 \%$. For Model-2, the computation time is improved 2.58 times faster than that with 4 processors and required memory is reduced by $34.8 \%$. As for the size of required memory, these results show an improvement better than that described in the previous section (30\% for $2-16$ and $47 \%$ for $4-16$ ). On the other hand, in the case of Model-2, although the number of processors is increased 4 times, the reason the computation time is not so improved (2.58 times) is that the time taken for calculation of the transformation matrix, which is not parallelized and is performed on the server program, is lengthened. An actual computation time taken by the server program is an average of 14 seconds, and is $35 \%$ of the total time taken for one iteration.

Figures 11 and 12 show the alignment results of the Great Buddha of Nara and Bayon in Cambodia, respectively. It takes approximately 5 minutes for 20 iterations for Model-1 and approximately 15 minutes for Model-2.

\section{Conclusion}

In this paper, we have proposed the parallel method for simultaneous alignment of multiple range images. In considering time performance and memory performance, we parallelized the alignment algorithm. Then, we implemented this method on a PC cluster, and showed its validity by aligning a large number of range images simultaneously. Future work will deal with accelerating the computation of transformation matrices.

\section{Acknowledgment}

This work is supported by Ikeuchi CREST (Core Research for Evolutional Science and Technology) of JST (Japan Science and Technology Corporation). The author would like to thanks the staffs of Todaiji Temple in Nara, Japan and Koutokuin in Kamakura, Japan. The Bayon in Cambodia was digitized with the cooperation of Japanese Government Team for Safeguarding Angkor (JSA). 


\section{References}

[1] K. Ikeuchi and Y. Sato, Modeling from Reality, Kluwer Academic Press, 2001.

[2] K. Ikeuchi, Modeling from Reality, In Proc. Third International Conference on $3 D$ Digital Imaging and Modeling (3DIM), Quebec City, Canada. May 28- June 1, 2001

[3] M. Levoy et al. The Digital Michelangelo Project. In Proc. SIGGRAPH 2000, pp.131-144.

[4] Greg Turk and Marc Levoy. Zippered polygon meshes from range images. In Proc. SIGGRAPH '94, pp. 311-318. ACM, 1994.

[5] Curless, B., Levoy,M., A Volumetric Method for Building Complex Models from Range Images, In Proc. SIGGRAPH' '96, ACM, 1996, pp. 303-312.

[6] M. Wheeler, Y. Sato, and K. Ikeuchi, Consensus surfaces for modeling $3 \mathrm{D}$ objects from multiple range images, In Proc. IEEE International Conference on Computer Vision, pp.917-923, Jan, 1997.

[7] R. Sagawa, K. Nishino, K. Ikeuchi, "Robust and Adaptive Integration of Multiple Range Images with Photometric Attributes", In Proc. IEEE Computer Society Conference on Computer Vision and Pattern Recognition, vol.2, pp.172-179, 2001

[8] P. J. Besl and N. D. McKay, A method for registration of 3-D shapes, IEEE Transactions on Pattern Analysis and Machine Intelligence, 14(2) 1992, 239-256.

[9] Y. Chen and G. Medioni, Object modeling by registration of multiple range images, Image and Vision Computing 10(3), 1992, 145-155.

[10] Blais, G. and Levine, M. "Registering Multiview Range Data to Create 3D Computer Objects," IEEE Transactions on Pattern Analysis and Machine Intelligence, Vol. 17, No. 8, 1995.

[11] T. Masuda, K. Sakaue, and N. Yokoya, Registration and Integration of Multiple Range Images for 3-D Model Construction. In Proc. Computer Society Conference on Computer Vision and Pattern Recognition, 1996.

[12] P. J. Neugebauer. Reconstruction of Real-World Objects via Simultaneous Registration and Robust Combination of Multiple Range Images. International Journal of Shape Modeling, 3(1\&2):71-90, 1997.

[13] R. Bergevin, M. Soucy, H. Gagnon, and D. Laurendeau. To-wards a general multi-view registration technique. IEEE Transactions on Pattern Analysis and Machine Intelligence, 18(5):540-547, May 1996.

[14] R. Benjemaa and F. Schmitt. Fast global registration of 3d sampled surfaces using a multi-z-buffer technique. In Proc. Int. Conf. on Recent Advances in 3-D Digital Imaging and Modeling, pages 113-120, May 1997.

[15] Z. Zhang. Iterative point matching for registration of free-form curves and surfaces. International Journal of Computer Vision, 13(2):119-152, 1994.

[16] David A. Simon, Martial Hebert, and Takeo Kanade. Realtime 3-D pose estimation using a high-speed range sensor. In IEEE Intl. Conf. Robotics and Automation, pages 2235-2241,San Diego, California, May 8-13 1994.

[17] Langis, C., Greenspan, M., and Godin, G.. The parallel iterative closest point algorithm. In Proc. International Conference on 3D Digital Imaging and Modeling (3DIM), Quebec City, Quebec. May 28- June 1, 2001.

[18] M. Garey, D. S. Johnson, Computers and Intractability: A Guide to the Theory of NP-Completeness, San Francisco, CA: Freeman, 1979.

[19] Alex Pothen, Horst D. Simon, and Kang-Pu Liou. Partitioning sparse matrices with eigenvectors of graphs. SIAM Journal of Matrix Analysis and Applications, 11(3):430-452, 1990.

[20] L. Hagen, A. B. Kahng, "New Spectral Methods for Ratio Cut Partitioning and Clustering", IEEE Transactions on Computer-Aided Design, Vol. 11, No. 9, pp. 1074-1085, September, 1992.

[21] G. Karypis and V. Kumar. A fast and high quality multilevel scheme for partitioning irregular graphs. SIAM Journal on Scientific Computing, 20(1):359-392, 1998.

[22] S. Hauck and G. Borriello, "An Evaluation of Bipartitioning Techniques", IEEE Transactions on ComputerAided Design of Integrated Circuits and Systems, Vol. 16, No. 8, pp. 849-866, August 1997.

[23] A. George and J. W.-H. Liu. Computer Solution of Large Sparse Positive Definite Systems. Prentice-Hall, Englewood Cliffs, NJ, 1981.

[24] B. W. Kernighan, S. Lin, "An Efficient Heuristic Procedure for Partitioning of Electrical Circuits", Bell Systems Technical Journal, Vol. 49, No. 2, pp. 291- 307, February 1970.

[25] C. M. Fiduccia, R. M. Mattheyses, "A Linear-Time Heuristic for Improved Network Partitions", Design Automation Conference, pp. 241-247, 1982.

[26] B. Krishnamurthy, "An Improved Min-Cut Algorithm for Partitioning VLSI Networks", IEEE Transactions on Computers, Vol. C-33, No. 5, pp. 438-446, May 1984.

[27] http://www.cyra.com. 